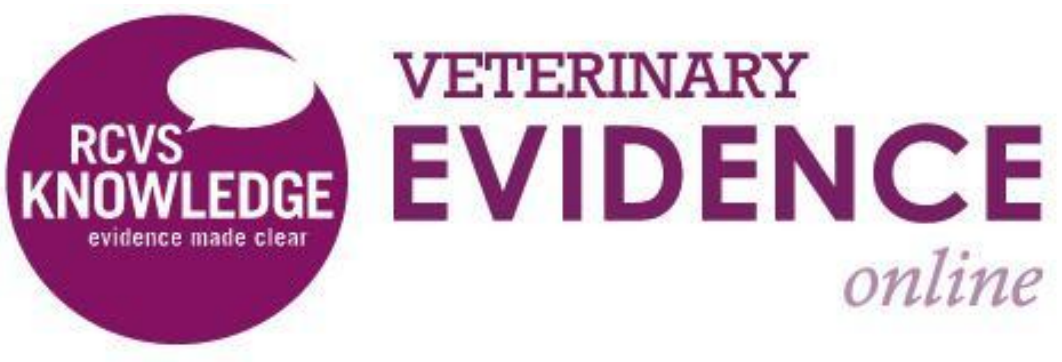

\title{
Evaluation of Achilles Tendon Injuries With Findings From Diagnostic Musculoskeletal Ultrasound in Canines - 43 Cases
}

Lauri-Jo Gamble DVM, CCRP ${ }^{1}$

Debra A. Canapp DVM, CCRT, CVA, DACVSMR ${ }^{1}$

Sherman O. Canapp DVM, MS, CCRT, DACVS, DACVSMR ${ }^{1^{*}}$

${ }^{1}$ Veterinary Orthopedic and Sports Medicine, 10975 Guilford Rd, Annapolis Junction, MD 20701, USA
${ }^{*}$ Corresponding Author (scanapp@vosm.com)

ISSN: 2396-9776

Published: 12 Aug 2017

in: Vol 2, Issue 3

DOI: http://dx.doi.org/10.18849/ve.v2i3.92

Reviewed by: Alycen Lundberg (DVM) and Stephen Jones

(MVB, MS, DACVS) 
Objective: The objective was to describe clinical and ultrasonographic characteristics of canine Achilles tendon injuries.

Background: Even though there have been publications describing characteristics of the normal and injured Achilles tendon by diagnostic ultrasound, there has been no previous in-depth ultrasonic evaluation and characterisation of Achilles tendon strains.

Evidentiary value: This is a retrospective case series report of 43 dogs that underwent musculoskeletal diagnostic ultrasound evaluation of their strained Achilles tendons, with and without surgical repair.

Methods: Data was collected from medical records of patients from August 2009 to May 2016. Inclusion criteria for dogs included a diagnosis of Achilles tendon injury based on the presence of fibre pattern modification within one or more components of the Achilles mechanism on initial diagnostic ultrasound.

Results: It was found on the diagnostic ultrasound that Achilles tendon injuries were strictly unilateral in 51.2\% ( $n=22 / 43$ ) of cases and bilateral on presentation in $48.8 \%(n=21 / 43)$ of cases. $18.2 \%$ of the unilateral case progressively became bilateral over time $(n=4 / 22)$. The injury was partial in $90.7 \%(n=39 / 43)$ of the cases and all three components of the Achilles mechanism were completely disrupted in only $9.3 \%$ of the cases $(n=4 / 43)$. The partial injuries incorporated changes within the fibre patterns of all 3 components of the Achilles in $53.8 \%$ $(n=21 / 39)$ of the cases and alteration of both the gastrocnemius tendon (GT) and common tendon (CT) in $41.0 \%(n=16 / 39)$ of the cases. Grade III strains were predominant $(44.3 \%)$, followed by grade I $(28.6 \%)$ and grade II (27.1\%). Damage occurred most commonly at the tendino-osseous insertion (77\%), with injury occurring less frequently diffusely along the body of the tendon $(12.5 \%)$ or at the musculotendinous junction (10.5\%).

Conclusion: Diagnostic musculoskeletal ultrasound was used to identify lesions of Achilles tendon consistent with different grade of strain, as well as identifying the specific region of injury. Diagnostic ultrasound findings were correlated to the surgical findings and previous literature.

Application: Diagnostic musculoskeletal ultrasound provides a non-invasive diagnostic modality for patients suspected of having Achilles tendon strain. Further investigation is needed to establish treatment protocols based on ultrasonographic 3-tier grading scheme for Achilles tendon strain injury.

Abbreviations: Common tendon (CT), computed tomography scan (CT scan), gastrocnemius tendon (GT), magnetic resonance imaging (MRI), myotendinous junction (MTJ), superficial digital flexor tendon (SDFT).

\section{INTRODUCTION}

The Achilles tendon, or common calcanean tendon, is made of three components (three separate tendons made of the tendinous insertion of five muscles attaching to the calcaneal tuberosity): (1) the gastrocnemius tendon (GT), (2) the superficial digital flexor tendon (SDFT) and (3-5) the common tendon (CT, combined tendons of gracilis, semitendinosus and biceps femoris) (Evans et al., 2013; Johnson, 2005). The GT is responsible for tarsal extension and inserts on the proximal dorsal surface of the calcaneus. The SDFT inserts 
collaterally, through the superficial digital flexor retinaculum, at the proximal calcaneus before continuing distally and attaching to the second row of the phalanges. The last component, the CT, inserts at the medial aspect of the calcaneus, but plays a minor role in tarsal extension (Evans et al., 2013).

Although Achilles tendon rupture is an uncommon tendon injury, it has been described frequently in dogs (Reinke et al., 1993; King et al., 2003; Corr et al., 2010; Spinella et al., 2010). Mature middle-aged, medium to large-breed active dogs seem to be most commonly affected by this type of injury (Kramer et al., 2001; Harasen, 2006; Rivers, 1997).

Disruption of the Achilles tendon can be complete (all components, GT, SDFT, CT) or partial (leaving notably the SDFT intact). Meutstege proposed in 1993 a classification for canine Achilles tendon injuries based on the localisation of lesions and gross pathology, where type I is a complete rupture, type lla affects the musculotendinous junction, type IIb has an intact paratendon, type IIc is a GT avulsion with intact SDFT and type III tendinosis and/or peritendinitis (Meutstege, 1993). When there is a complete disruption of the Achilles tendon apparatus the dog will be initially non-weight bearing and a swelling may be noted at the point of the tarsus (calcaneus) (Caine et al., 2009; Cervi et al., 2010; Spinella et al., 2010). In some cases, a skin lesion/laceration may be evident and there may be a palpable tendon defect (Vaughan, 1981). With more chronic lesions, the hock will sink to a hyperflexed position with associated hyperextension of the knee and plantigrade stance with the metatarsals and digits lying flat on the floor (walking on the soles of the paws; flat footed) (Johnson, 2005; Meutstege, 1993; King et al., 2003). For a partial tear or lengthening of the Achilles tendon system, the toes are curled downward (crab claw stance; digital knuckling) (Johnson, 2005; Meutstege, 1993). Dogs with disruption only affecting the GT and CT display a hyperflexed tarsus with contracture of the toes since the intact SDFT is placed under considerable tension, which pulls on its insertions on the palmar surface of the proximal end of the middle phalanges of digits II, III, IV, and V, contracting the toes (Johnson, 2005; Meutstege, 1993).

Achilles tendon rupture in the literature tends to be associated with an acute traumatic episode: either an impact injury resulting in avulsion of the tendon from the calcaneus or a direct sharp trauma to the musculotendinous unit (cut, laceration) (Cervi et al., 2010; Baltzer et al., 2009). Chronic degeneration of the Achilles tendon is also possible and is more frequently seen in Labrador Retrievers and Doberman Pinschers (Harasen, 2006; Meutstege, 1993; Morton et al., 2015). Active dogs can also have an acute on chronic presentation (chronic degeneration exacerbated by an acute injury). Degenerative injuries may also be secondary to other causes such as systemic disease (Spinella et al., 2010) (i.e. Cushing's disease) or iatrogenic etiologies (Shakibaei et al., 2001; Lim et al., 2008; Hossain et al., 2008; Baltzer et al., 2009) (steroid administration, fluoroquinolone treatment) (Shakibaei et al., 2001).

Ultrasound evaluation has become a valuable and practical tool in assessing the Achilles tendon. It is used frequently in human medicine for diagnostic evaluation and grading of Achilles tendon injuries (Griffith, 2014; Luscombe et al., 2003; Henderson et al., 2015; Royall, 2011; Maffulli et al., 2004). Another advantage of musculoskeletal ultrasound is the possibility to use the contralateral limb as a comparison to evaluate symmetry of the tendon. This comparison may be difficult in the presence of bilateral conditions, but the ultrasonographic findings often differ between limbs and therefore asymmetrical findings within both Achilles tendons are notable. Sedation and anaesthesia are not necessary which makes it of benefit over CT scan and MRI. Sonographic evaluation is also less expensive, making it a more viable option for both initial diagnostics and recheck examinations. 
Ultrasonographic evaluation of the normal Achilles tendon was first reported in 1997 by Kramer (Kramer et al., 1997) as part of a report investigating the utility of ultrasonography as a diagnostic imaging procedure in orthopaedic problems in small animals. A more specific study was repeated in 2005 by Lamb (Lamb et al., 2005) where detailed evaluation of the canine Achilles tendon was performed on greyhound cadavers compared to dissected specimens. A few years later, characterisation of the soft tissue structures of the canine tarsus in medium to large breed dogs was obtained by Caine (Caine et al., 2009). An additional ultrasonic study has been performed by Rivers (Rivers, 1997) to preoperatively evaluate canine Achilles tendon injury in a series of six cases with surgical confirmation of the diagnosis. It was found that ultrasonographic examination could not differentiate partial from complete Achilles tendon rupture based on sonographic echotexture pattern (Rivers, 1997). However, it was later established in 2001 by Kramer (Kramer et al., 2001) that ultrasonography allowed identification and differentiation of total ruptures and the differentiation of partial ruptures into deep or superficial ruptures, or those comprising muscular tears. Additionally, a few case reports on Achilles tendon injury and its treatments in dogs have mentioned successful use of diagnostic ultrasound (Baltzer et al., 2009; Case et al., 2013; Mueller et al., 2009; Spinella et al., 2010).

The aim of this retrospective case series was to report and characterise the ultrasonic findings of Achilles tendon injuries in the general canine population seen at one referral centre.

\section{RESULTS}

\section{Population Sample}

Most of the 43 patients included in this case series were strictly companion animals ( $n=36 / 43 ; 83.7 \%)$. The seven other patients had a listed occupation or hobby including field trial/hunting $(n=4)$, police/security $(n=1)$, conformation $(n=1)$, and a combination of multiple activities including agility $(n=1)$. Excluding mixed breeds, 18 dog breeds were evaluated. The most common were Labrador $(n=11 ; 25.6 \%)$, Doberman $(n=6 ; 14.0 \%)$ and Golden retrievers $(n=3 ; 7.0 \%)$. Females were overly represented, but this tendency was not significant with $55.8 \%$ of the patients being female ( 22 spayed, 2 intact), and $44.2 \%$ being male ( 13 neutered, 6 intact). The mean and the median age at the time of initial presentation were 6 years and 3 months and 7 years respectively (range 5.6 months to 12 years and 3 months). The mean body weight was 66.0 pounds (30 kg) with a median body weight of 62.4 pounds $(28.4 \mathrm{~kg}$ ) (range of $12.3-150$ pounds). The vast majority ( $\mathrm{n}=37 / 43$; $86.0 \%)$ of the patients in this case study had a chronic presentation which was defined by a persistence of clinical signs over more than one week. However, the mean estimated time to presentation was 6 months with 6 cases even having a history greater than one year.

\section{Initial Orthopaedic Examination}

Visible lameness was reported in 35 of the 43 patients (81.4\%) which was subjectively graded on a I to VI scale (Table 2) as grade I for 5 patients, grade II for 14 patients, grade III for 5 patients, grade IV for 6 patients and grade $\mathrm{V}$ for 5 patients. Shorter stride was also noted in 2 cases. Standing and walking stance were characterised by a hyperflexed tarsus ("dropped") due to a rupture of the Achilles tendon in $34.9 \%$ ( $n=15 / 43$ ), whereas the classic contracture of the digits ("claw stance") was present $41.9 \%$ of the time $(n=18 / 43)$. The 
reported plantigrade stance ("flat footed") (Spinella et al., 2010; Corr et al., 2010; Baltzer et al., 2009) was not as frequently expressed; it was only visible in $7.0 \%$ of the patients $(n=3 / 43)$. The most reliable findings on physical examination appeared to be moderate to severe soft tissue swelling at the insertion point of the Achilles tendon to the calcaneus (point of the hock) since it was noted $81.4 \%$ of the time ( $n=35 / 43$ ). Perception of generalised or partial thickening of the Achilles tendon was not always present with only $37.2 \%$ of the patients exhibiting this characteristic $(n=16 / 43)$. Evidence of wounds and laceration was only reported for 2 patients (4.7\%). Discomfort upon palpation of the Achilles tendon and/or during flexion of the tarsus was evident in $32.6 \%(n=14 / 43)$.

On physical examination, it was suspected that Achilles tendon was injured unilaterally $79.1 \%$ of the cases $(n=$ $34 / 43,13$ right, 21 left) and bilaterally in $20.9 \%$ of the cases ( $n=9 / 43)$. No grading of the strain was attempted only based on physical examination and clinical assessment.

\section{Radiographs}

Radiographs were unremarkable for two cases (4.7\%). Radiographs of the affected tarsus of all the other 41 patients revealed soft tissue swelling at the insertion point of the Achilles tendon, as illustrated in figure 1 . This change was mild in 4 cases (9.8\%) compared to moderate to severe in 37 cases (90.2\%). Mild bone remodeling and periosteal reaction was noted along the calcaneal tuberosity at the insertion point of the Achilles tendon in 11 cases (26.8\%, see figure 1 A.). Calcification or dystrophic mineralisation was noted within 8 Achilles tendons $(19.5 \%$, see figure $1 \mathrm{~A})$ whereas bone fragment and suspected avulsion of the calcaneus was present in another 8 cases (19.5\%, see figure $1 \mathrm{~B}$ and C). Signs compatible with tarsal degenerative joint disease were seen in 2 cases (4.9\%) including changes in shape and opacity of the joint margins, osteophyte formation, illdefined subchondral bone/cartilage interface. Previous implant migration/failure was noted in 2 cases (4.9\%).

\section{Figure 1 - Tarsal Radiographs of Different Patients Affected by Achilles Tendon Injury}

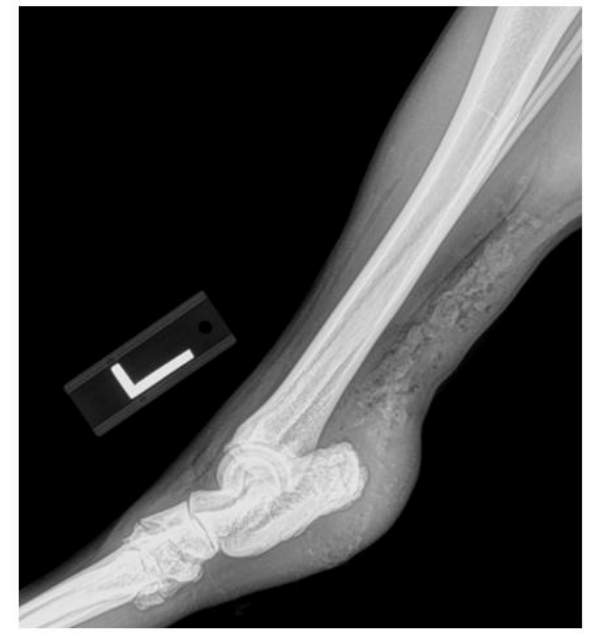

A

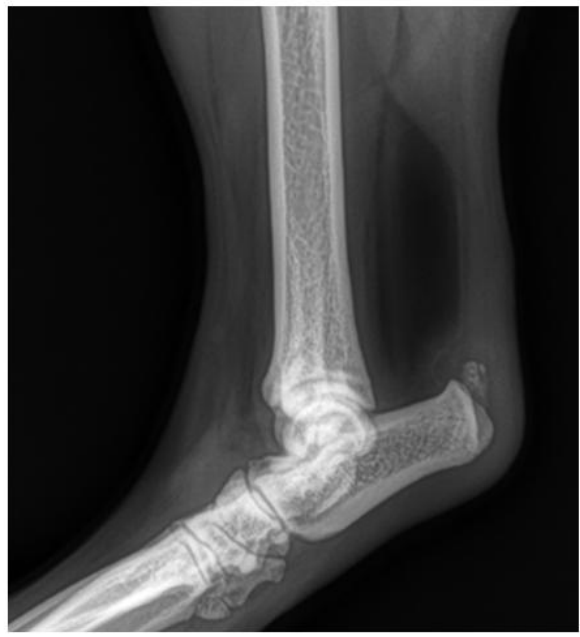

B

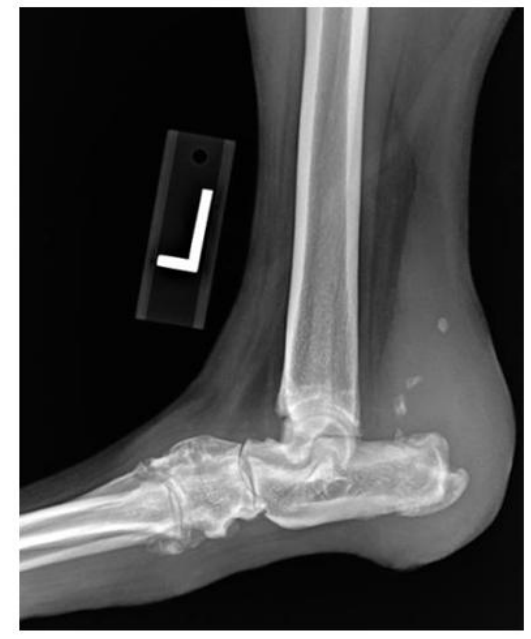

C

\section{A- Hyperextended latero-medial view left tarsus}

Moderate to severe soft tissue swelling was noted at the insertion point of the Achilles' tendon. Mild remodeling was present along the calcaneus at the insertion point of the Achilles' tendon, mild calcification was also noted within the tendon. 
B- Routine latero-medial view left tarsus Avulsion of Achilles tendon. Moderate soft tissue swelling was present at the insertion point of the Achilles tendon on the calcaneus. Bone chip presence is indicative of a presumptive avulsion injury.

\section{C- Routine latero-medial view left tarsus}

Severe soft tissue swelling was observed at the insert point of the Achilles tendon on the calcaneus. Bone chips and mineralisation were present, indicating a presumptive chronic avulsion injury.

\section{Musculoskeletal Diagnostic Ultrasound}

Compared to the initial physical examination, it was observed on the diagnostic ultrasound that Achilles tendon injuries were unilateral in $51.2 \%$ of cases ( $n=22 / 43,7$ right, 15 left) and bilateral on presentation in $48.8 \%$ of cases $(n=21 / 43)$. This is significantly $(p<0.05)$ different from the observed clinical signs on physical examination; where lameness, swelling or hyperflexion of the hock were noted unilaterally in $79.1 \%$ of the cases ( $n=34 / 43,13$ right, 21 left) and bilaterally in $20.9 \%$ of the cases ( $n=9 / 43)$. During the available follow-up period (6-36 months), 4 of 22 unilateral cases (18.2\%) progressively start showing visible ultrasonographic changes on the contralateral Achilles tendon.

The injury was partial in $90.7 \%$ of the cases $(n=39 / 43$ ) and all three components of the Achilles mechanism were completely disrupted in the remaining $9.3 \%$ of the cases $(n=4 / 43)$. However, changes within the fibre patterns of all 3 components of the Achilles were seen in $53.8 \%$ of the dogs affected by partial Achilles tendon injury ( $n=21 / 39)$. In some cases $(n=4)$, the SDFT was still intact but the fibres were severely affected at the same level of the complete disruption of the GT and CT. The "claw stance" posture was significantly more prevalent $(p<0.05)$ with intact SDFT, but was also seen in one case with a complete tear of the Achilles mechanism. Disruption of only the GT and CT was noted in $41.0 \%$ of the cases ( $n=16 / 39$ ). See Table 1 for the complete repartition of the components of the Achilles tendon involved within the strain.

\begin{tabular}{|l|l|l|}
\hline Localisation & Percentage & $\mathbf{n = 6 4}$ \\
\hline Gastrocnemius tendon (GT) & $6.25 \%$ & 4 \\
\hline Common tendon (CT) & $17.19 \%$ & 11 \\
\hline Superficial digital flexor tendon (SDFT) & $4.69 \%$ & 3 \\
\hline GT + CT & $25.00 \%$ & 16 \\
\hline GT + SDFT & $1.56 \%$ & 1 \\
\hline CT + SDFT & $6.25 \%$ & 4 \\
\hline All components & $39.06 \%$ & 25 \\
\hline
\end{tabular}

Table 1: Achilles Tendon Injuries Localisation noted on Diagnostic Ultrasound

*This table includes only major findings (clinically relevant) for each patient, but may include changes of bilateral Achilles tendons. A total of 64 Achilles tendons with abnormalities were evaluated. When different components were involved, the grade may have been different for each component. 
Achilles tendon strains were graded on a scale of I-III (Table 3). Grade III strains were predominant (44.3\%), followed by grade I (28.6\%), and grade II (27.1\%). Grade I strains (see figure 2 ) had intact architecture, but thickening, inflammation, and stretching may be visible. Grade II strains (see figure 3) were characterised by partial disruption of the fiber alignment, laxity and hypoechoic fibres. Grade III strains (see figure 4), or complete disruption, were distinguished by the presence of a hypoechoic band bordered by the retracted hyperechoic tendon ends. See supplementary Table 1 for the complete list of the ultrasonic changes noted and refer to Figures 2-4 for illustration of those changes. The strain grade was not correlated to sex, breed, age, occupation and level of activity $(p<0.05)$.

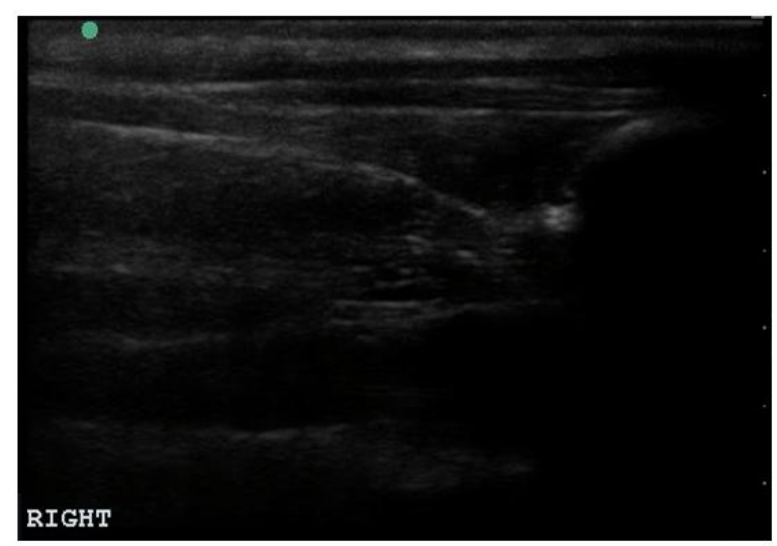

A

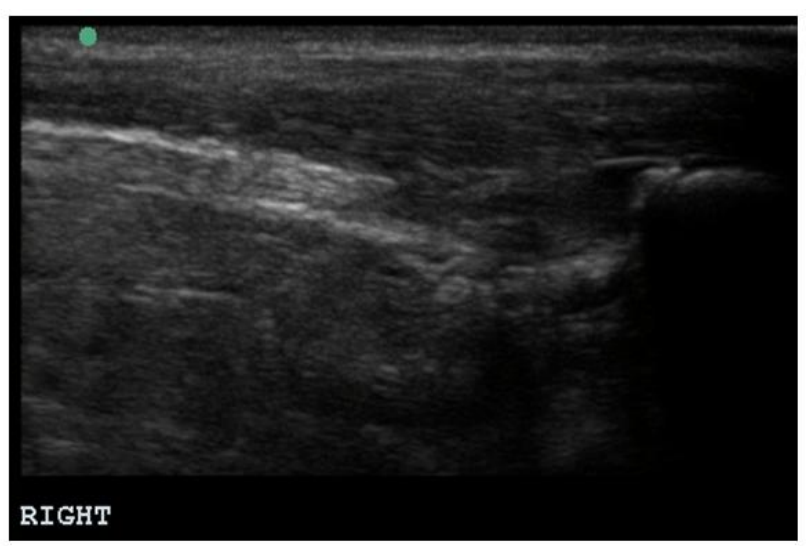

B

Figure 2: Grade I Strain

\section{A- Longitudinal view of grade I strain of common tendon}

Evidence of right CT early disruption with mild hyperechoic/hypoechoic foci noted at the point of insertion.

\section{B- Longitudinal view of grade I strain of common and gastrocnemius tendons}

There was a generalised hypoechoic fibre pattern to the CT and GT with no disruption noted. In addition the common tendon bursa also showed chronic hyperechoic changes consistent with bursitis. 


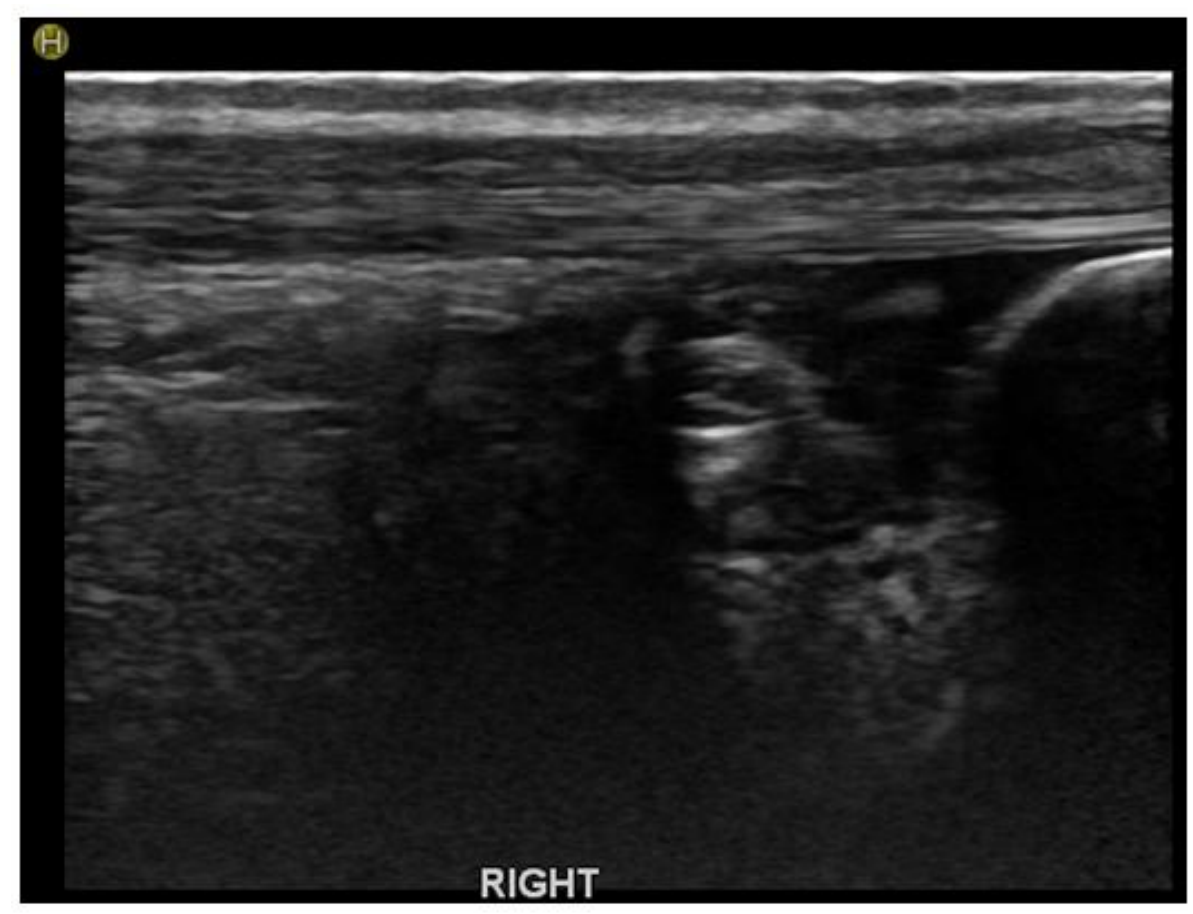

Figure 3: Grade II Strain

Longitudinal view grade II strain gastrocnemius and common tendons with grade I superficial digital flexor tendon

The right SDFT appeared slightly thickened but showed overall good pattern. There was a mild amount of periosteal hyperechoic changes noted at the deep margin of the calcaneus. There was moderate disruption of the GT, mid tendon with normal identifiable pattern at the distal 1/3 of the tendon and more disruption note proximal to that point. The CT was moderately disrupted at the point of insertion with severe hypoechoic fib pattern and hyperechoic foci at the point of insertion at the calcaneus. In addition there were moderate areas of anechoic findings, indicating moderate fluid accumulation within the bursa.

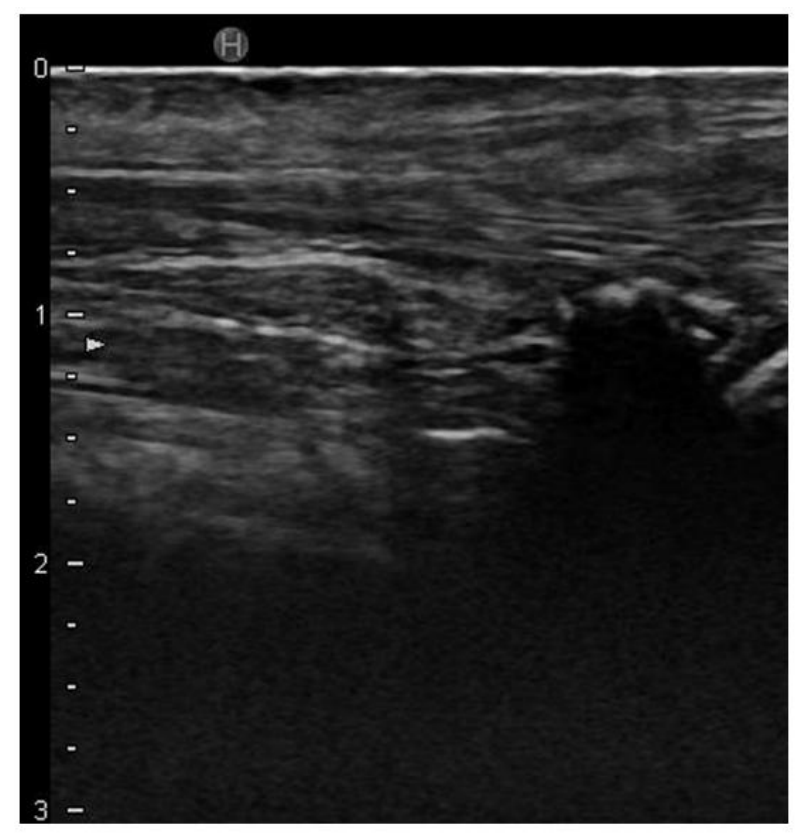

A

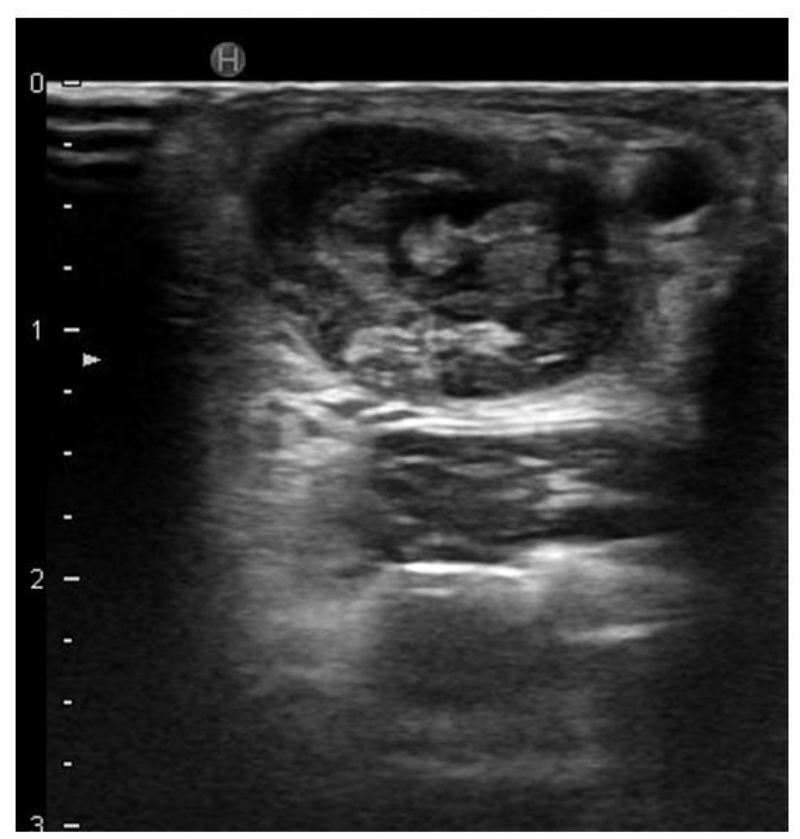

B 
Figure 4: Grade III Strain

A- Longitudinal view of a grade III strain of the Achilles tendon (all components involved)

This Achilles tendon was hypoechoic with loss of normal and mottled appearance. There was a significant amount of hypoechoic soft tissue changes with lack of fibre pattern noted superficially at and just proximal to the calcaneus. The calcaneal tuberosity also had visible periosteal reaction.

B- Cross-sectional view of a grade III strain of the Achilles tendon (all components involved)

There was severe disruption of the GT with a minor amount of identifiable fibre pattern at the distal $1 / 3$ of the tendon, attaching to the calcaneus. There were also age/degenerative hyperechoic changes noted within the common tendon bursa. In general the areas of loss of fibre pattern showed hypoechoic tissue with some fibrous tissue.

The vast majority of the injuries evaluated in these cases were chronic, hence hyperechoic foci, mottled or focal hypoechoic core lesions, overall thickening of the tendon, mineralisation, periosteal hyperechoic reaction along the calcaneus in addition to calcified remodelling were noted. In the chronic cases, a significant amount of hypoechoic soft tissue swelling was also noted within the area of the calcaneus insertion making visualisation of the SDFT more difficult. More acute injuries would differ by the presence of oedema (hypoechogenicity between the fibres) and/or significant amount of vascularisation in the disrupted area. Generalised hypoechoic appearance to the muscle belly indicated a muscle fibre injury and bruising. In comparison, muscle atrophy was characterised by a more hyperechoic fibre pattern. The term laceration was not used in any diagnostic ultrasound report. Therefore, this lesion was not characterised in this study.

Damage occurred most commonly at the tendino-osseous insertion (77\%) with visible hypoechoic fibre lesions and fibre disruption. The MTJ was affected less frequently with injury occurring in $10.5 \%$ of the cases and was more likely to be observed in animals that exhibited a plantigrade stance. In $12.5 \%$ of the cases, the disruption and loss of fibre pattern noted was more generalised and diffuse along the body of the tendon starting at the insertion onto the calcaneus all the way to the MTJ. For one patient, this change was accompanied by several hyperechoic fibres "coiled up" amongst the altered muscle tissue which was most likely representing retracted tendon fibers in addition to a musculotendinous rupture (see figure 5). Those ultrasonographic characteristics were noted in a patient affected by a complete disruption of the Achilles mechanism. 


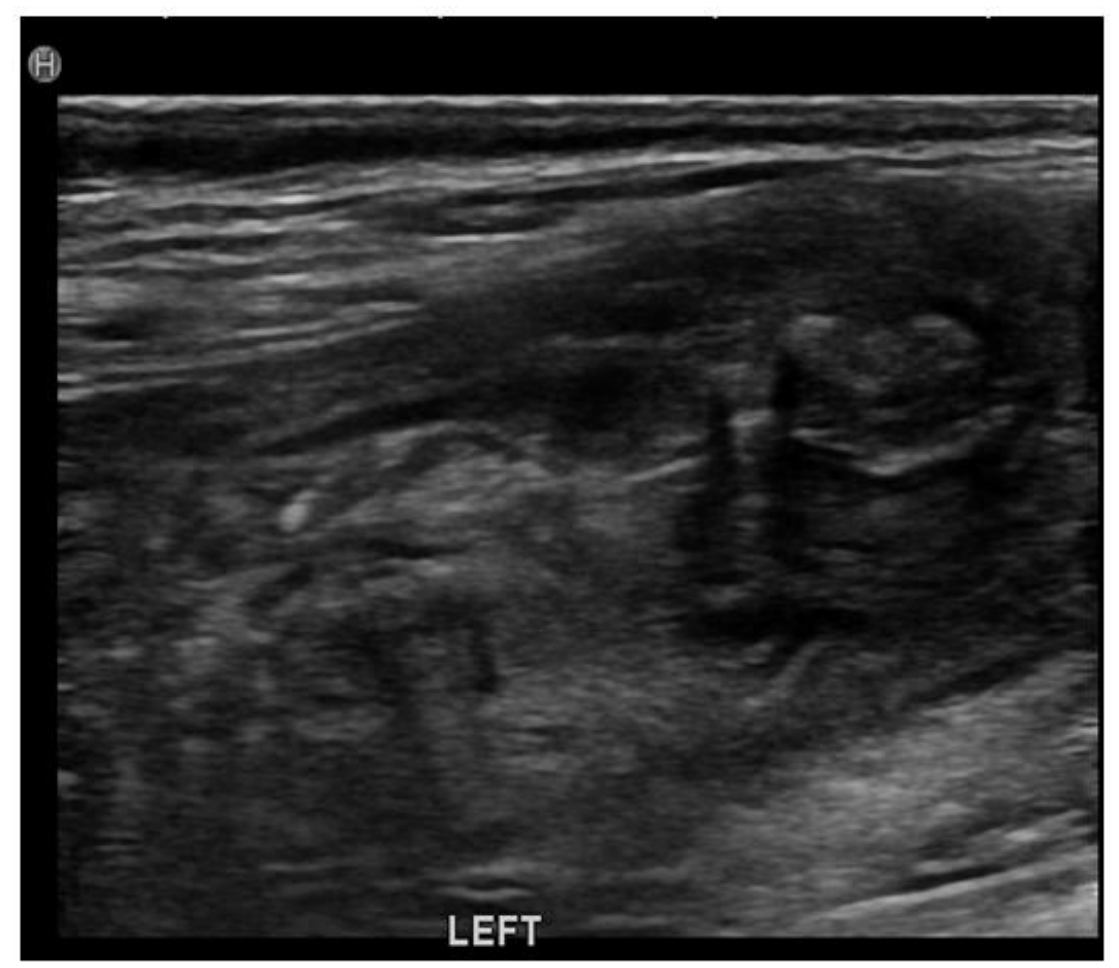

Figure 5: Alteration of the Musculotendinous Junction (MTJ)

Longitudinal view of the musculotendinous junction

The musculotendinous junction of both the GT and the SDF were significantly altered with lack of normal muscle of fibre tendon. Several hyperechoic fibs were "coiled up" amongst the altered muscle tissue which may be representing retracted tendon fibs from a distal rupture in addition to a MTJ rupture.

Evaluation of the bursa was performed while investigating the insertion of the common tendon. Mild amount of hyperechoic changes within the common tendon bursa was frequently identified as normal age-related changes (see figure $4 \mathrm{~B}$ ). Minor hyperechoic fibrous changes within the tendons were also typically agerelated. A diagnosis of bursitis was indicated by the presence of anechoic fluid at the point of insertion at the calcaneus along with calcification and fibrous changes within the bursa to a greater degree than the contralateral side, in addition to no other tendinous insertion fibre changes (see figures $2 \mathrm{~B}$ and 3 ).

\section{METHODS \& MATERIALS}

\section{Case Selection}

Medical records from August 2009 through May 2016 were analysed for canine and feline patients with suspected Achilles tendon injury assessed on physical examination (Veterinary Orthopedic Sports Medicine Group, Annapolis Junction, MD). Physical examination, diagnostic findings, ultrasonic findings, surgical findings, and treatment plans were reviewed. Data were collected for patients that underwent musculoskeletal diagnostic ultrasound evaluation of their Achilles tendons, with and without surgical repair. Inclusion criteria for patients included a diagnosis of Achilles tendon injury based on the presence of fibre pattern modification within one or more components of the Achilles tendon on initial diagnostic ultrasound. 
Patients were excluded from this study due to incomplete medical records, surgical repair performed without initial diagnostic ultrasound (even though follow-up ultrasounds were available), or absence of Achilles tendon pathology noted on diagnostic ultrasound. See supplementary Figures 1 and 2 for the description of the case selection process.

\section{Initial Orthopaedic Examination}

Complete orthopaedic examination, including gait assessment and palpation, was performed by ACVS board certified surgeons. Lameness was subjectively graded on a modified I to VI scale (Millis, 2004) (Table 2). Physical examination findings and the recognition of the classic gait - increased extension in the stifle with abnormal flexion in the tarsus with or without contracture of the toes or possibly plantigrade stance (Meutstege, 1993; Corr et al., 2010) associated with Achilles tendon injury were the first steps to diagnosing this condition.

\begin{tabular}{|l|l|}
\hline Grade of Lameness & Description \\
\hline I & Lameness is not perceptible at a walk but perceptible at a trot \\
\hline II & Lameness is perceptible at a walk and apparent at the trot \\
\hline III & Lameness is apparent at both a walk and trot \\
\hline IV & Lameness is apparent at a walk and severe to non-weight bearing at a trot \\
\hline V & Non-weight bearing lameness at a walk and trot \\
\hline VI & Unable to rise and walk \\
\hline
\end{tabular}

Table 2: Lameness Numeric Rating Scale

The integrity of the Achilles tendon was also tested in lateral recumbency; the tibiotarsal joint was flexed with the stifle held in an extended position (Meutstege, 1993). As previously described by Meutstege, when the hock was partially flexed while the stifle was extended and the digits contracted, it was considered consistent with an Achilles tendon tear with an intact superficial digital flexor tendon. When the hock was completely flexed while the stifle was extended without tension of the Achilles tendon, it was considered consistent with a complete Achilles tendon tear.

\section{Radiographs}

Routine flexed lateral and plantarodorsal radiographs ${ }^{\mathrm{a}}$ of the tarsus were performed in all 43 cases and evaluated by a board certified surgeon. Radiographs did not specifically aid in grading and characterisation of Achilles tendon strain since soft tissue injuries are not well appreciated using this modality, however indirect markers such as thickening or swelling of the tendon, mineralised fragments, or avulsion were noted. Swelling along insertion site on the calcaneus was subjectively graded as mild, moderate or severe by the same examiners. Although localisation of the injury to a particular soft tissue structure was not possible on radiographs, other tarsal pathologies including bone fractures, luxations (dislocation or misalignments of the tarsus), abnormal opening of a joint when stressed (varus, valgus, extension, flexion) were ruled out. 
Musculoskeletal diagnostic ultrasound of the Achilles tendon was offered to all patients presenting with suspected Achilles tendon injury. If elected, patients were placed in lateral recumbency and the tarsus and Achilles tendon were clipped and prepared routinely with the application of isopropyl alcohol and coupling gel to get direct contact and avoid artifact formation. Ultrasound of the affected calcaneal tendon was performed first with subsequent assessment of the contralateral unaffected tendon. Most examinations were performed by a single experienced practitioner (Debra A Cannap), except for 9 cases for which findings were all reviewed by DC. Sedation was not required to perform ultrasound examinations.

Ultrasound was performed using a Hitachi-Aloka Noblus system ${ }^{\mathrm{b}}(18 \mathrm{mHz}$ linear probe) and Sono Site system $^{c}(15 \mathrm{mHz}$ linear probe) to acquire longitudinal and cross-sectional images of the Achilles tendon and musculotendinous region. In static views, the tarsus was placed in a flexed position to provide some tension on the tendon. Dynamic ultrasonographic scanning, achieved by examining the tendon during flexion and extension of the tarsus, was also performed for optional diagnosis of complete Achilles tendon rupture. The transducer foot-print was a medium length to maintain good contact and proper alignment with the Achilles tendon. Echogenicity, fibre pattern, and size of each tendon were recorded and compared to the contralateral Achilles tendon. Every effort was made to keep the ultrasound beam perpendicular to the area of interest to avoid anisotropy. Anisotropy can result in the fibres of the tendon appearing hypoechoic when the angle of the tendon is no longer perpendicular to the ultrasound beam. This can occur when the ultrasound transducer is angled as little as 5 degrees off perpendicular (Griffith, 2014).

The anatomy was grouped into three components when approaching musculoskeletal ultrasound of the Achilles tendon: paired tendons of the gastrocnemius muscle (GT), common tendon (CT, combined tendon of the gracilis, semitendinosus and biceps femoris muscle), and tendon of the superficial digital flexor muscle (SDFT). Identification of tendon injuries was done using a modified 3-tier grading scheme (Archambault et al., 1998; Takebayashi et al, 1995) (Table 3). A partial injury was defined as the fibre pattern modification of one or more components of the Achilles tendon in comparison to a complete tear which involved the entire disruption of all three components (CT, GT, SDFT). With a partial injury, all three components may have been affected, but they could not all be grade III strain as this would have resulted in a complete tear. 


\begin{tabular}{|c|c|c|}
\hline Grade & Definition & Description \\
\hline I (mild) & $\begin{array}{l}\text { Overstretching of the tendon, } \\
\text { without a tear or loss of function }\end{array}$ & $\begin{array}{l}\text { - } \text { Simple stretch } \\
\text { - } \text { Thickening } \\
\text { - Inflammation } \\
\text { - Minimal structural involvement } \\
\text { - Microscopic tears, no fibre } \\
\text { disruption, intact architecture }\end{array}$ \\
\hline II (moderate) & $\begin{array}{l}\text { The general continuity of the tendon } \\
\text { is intact though its strength is } \\
\text { significantly reduced }\end{array}$ & $\begin{array}{ll}\text { - } & \text { Minor fibre disruption/partial } \\
\text { - } & \text { Minor/core lesion } \\
\text { - } & \text { Hypoechoic fibres } \\
\text { - Painful }\end{array}$ \\
\hline III (severe) & $\begin{array}{l}\text { Complete disruption, avulsion or } \\
\text { tearing of the tendon }\end{array}$ & $\begin{array}{ll}\text { - } & \text { Complete tear } \\
\text { - } & \text { Results in tendon dysfunction }\end{array}$ \\
\hline
\end{tabular}

Table 3: Definition and Description of Numerical 3-Tier Grading Scheme for Tendinous Strain Injury

An evaluation generally began with the probe placed longitudinally on the Achilles tendon in caudal/plantar approach. This view allowed for a general approach to the location to assess the anatomy (see figure 7A and 7B). A cross-sectional view was also of importance when evaluating the insertion of the Achilles tendon on the calcaneal tuberosity (see figure $7 C$ and 7D). The Achilles tendon was assessed for disruption of the fibre alignment, fibre tears, complete disruption, avulsion, mineralisation, or evidence of chronic fibrosis or bursitis. Careful evaluation of the region for concurrent tarsal collateral ligaments and retinaculum tear was pursued. The SDFT was dynamically assessed for luxation at the level of the calcaneus, as the hock was flexed. Following along all components of the Achilles tendon, the myotendinous junction (MTJ) and belly muscles were then also evaluated with the transducer placed longitudinally and cross-sectionally. With consideration for the treatment performed, diagnostic ultrasound recheck evaluations were typically recommended at 30,60 and 90 days to assess healing of the Achilles tendon.

\section{Statistics}

When indicated, non-parametric paired t-test was performedd. Significance was established at $p<0.05$. 


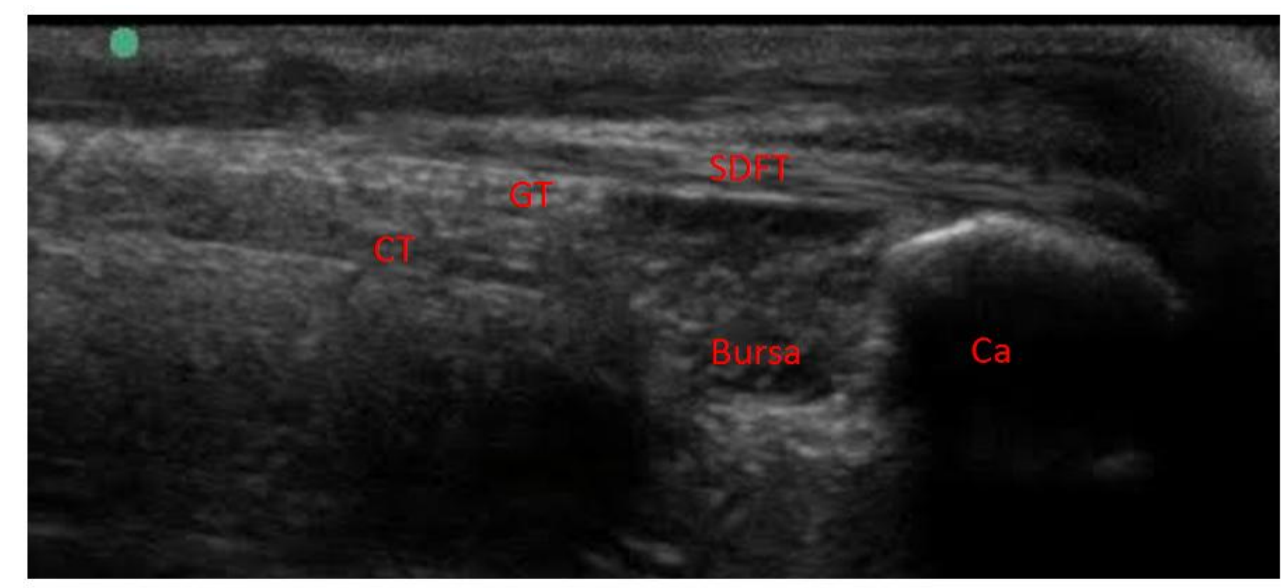

A

Figure 6A: Normal Achilles Longitudinal and Cross-Sectional Views Longitudinal view of a normal Achilles tendon

In longitudinal images of the distal part of the tendon the gastrocnemius tendon (GT) lies between the superficial digital flexor tendon (SDFT) and the common tendon (CT). The insertional bursa (Bursa) of the common tendon is visible at proximity of the calcaneal tuberosity ( $\mathrm{Ca}$ ) and its acoustic shadowing.

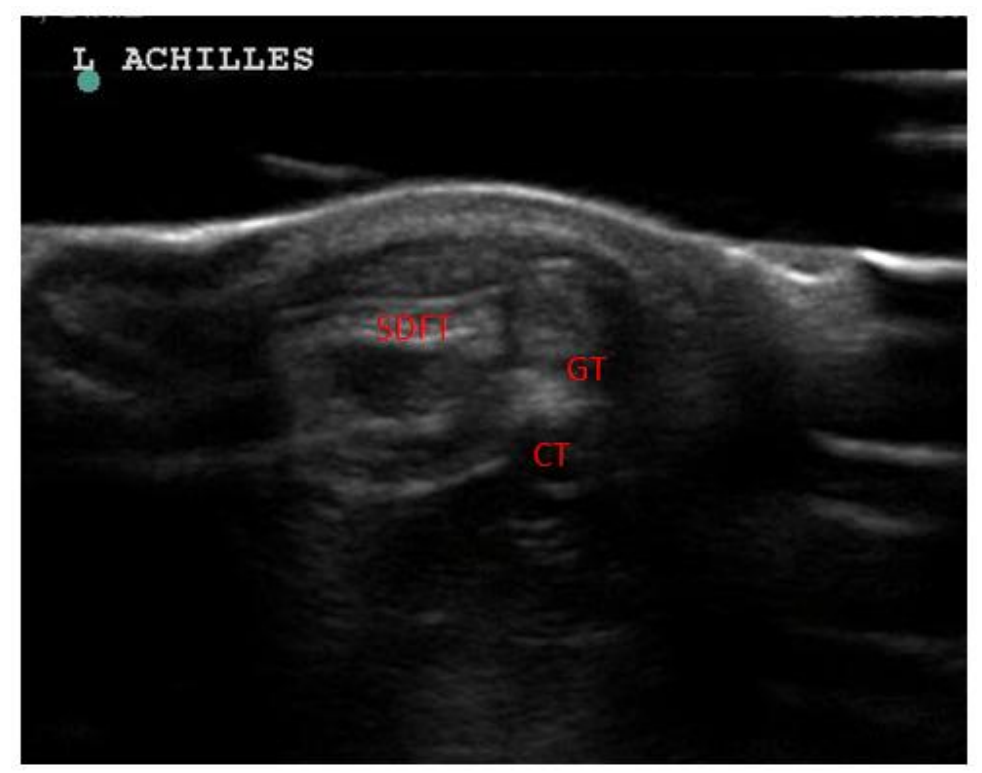

B

Figure 6B: Cross-sectional view of a normal Achilles tendon - proximal

In cross-sectional view, the appearance of the 3 components of the Achilles tendon remains virtually unchanged in the proximal and middle parts of the tendon. 


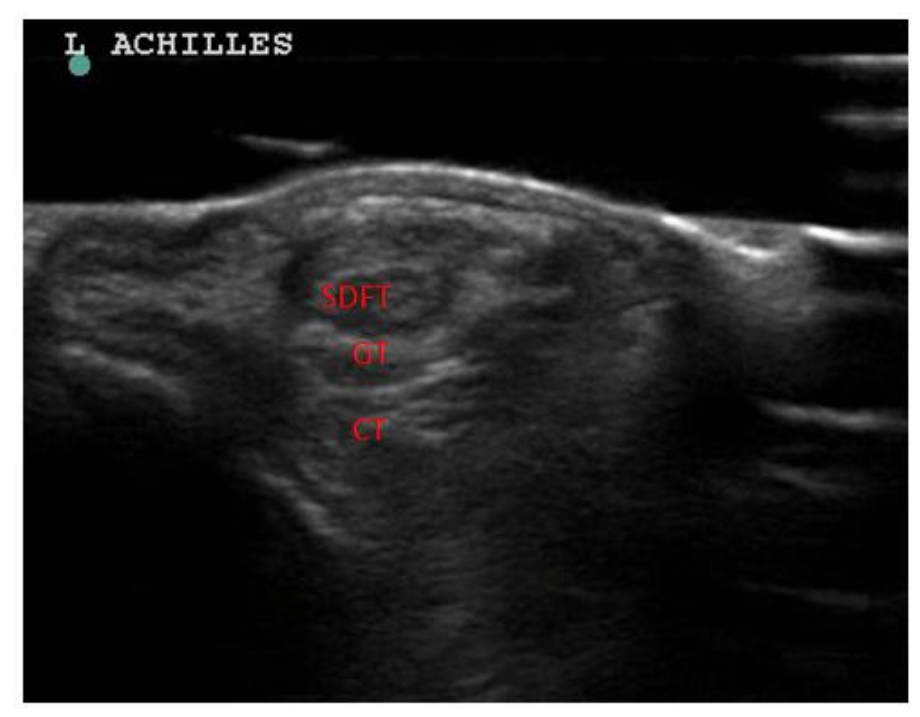

C

Figure 6C: Cross-sectional view of a normal Achilles tendon - distal

Toward the distal part, the superficial digital flexor tendon (SDFT) moves to a more superficial position.

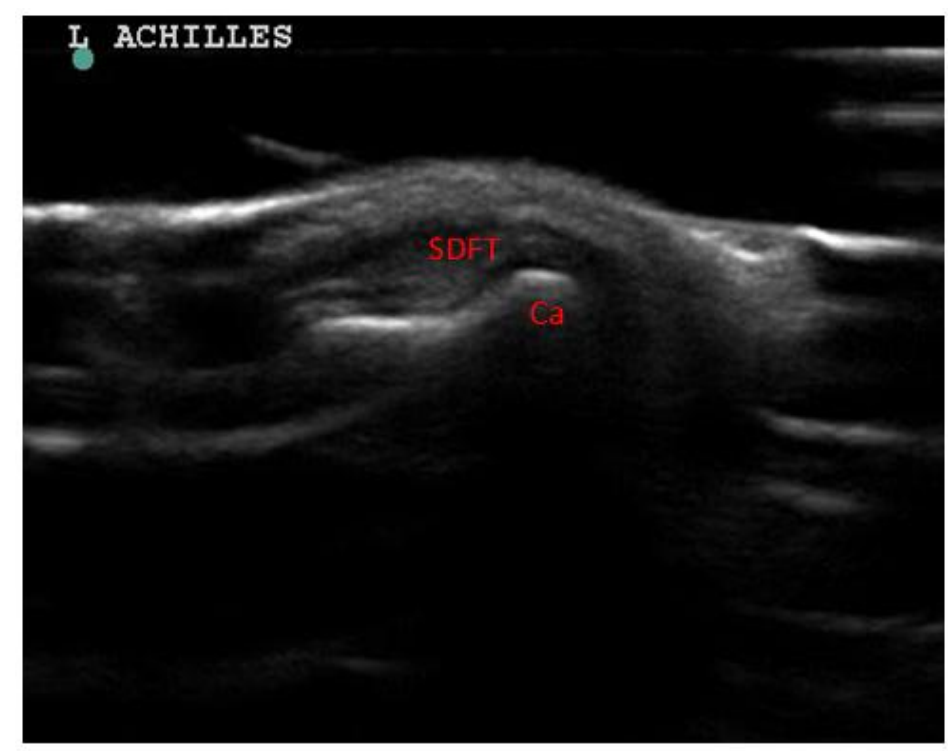

D

Figure 6D: Cross-sectional view of the SDFT

Visualization of the SDFT as it courses over the calcaneal tuberosity (Ca). 


\begin{tabular}{|c|c|c|c|c|}
\hline & GASTROCNEMIUS & $\begin{array}{l}\text { SUPERFICIAL } \\
\text { DIGITAL FLEXOR }\end{array}$ & COMMON TENDON & $\begin{array}{l}\text { LOCATION NOT } \\
\text { SPECIFIED or } \\
\text { GENERALISED }\end{array}$ \\
\hline $\begin{array}{l}\text { Hypoechoic Insertion Tendon } \\
\text { Lesion }\end{array}$ & 2 & 3 & 4 & \\
\hline Insertion Tendon Fibre Disruption & 35 & 8 & 33 & \\
\hline Core Lesion of MTJ & 4 & 3 & 11 & \\
\hline MJT Fibre Disruption & 5 & 4 & 3 & \\
\hline Hypoechoic Muscle Belly Lesion & 7 & 6 & 2 & 2 \\
\hline Muscle atrophy & 5 & 4 & & \\
\hline Avulsion & 5 & & 4 & 1 \\
\hline Thickening & 4 & 20 & 10 & \\
\hline Hyperechoic fibres & 8 & 5 & 26 & \\
\hline Hypoechoic fibres & 15 & 9 & 21 & \\
\hline Fibrosis & & 2 & 7 & \\
\hline Bursitis & & & 26 & \\
\hline Hypoechoic Soft Tissue Swelling & 11 & 7 & 6 & 24 \\
\hline $\begin{array}{l}\text { Hyperechoic Periosteal Reaction } \\
\text { along the Calcalneus }\end{array}$ & & & & 29 \\
\hline $\begin{array}{lll}\text { Calcified Remodeling and } \\
\text { Fragment near the Calcaneus }\end{array}$ & 5 & 1 & 4 & 11 \\
\hline Collateral Ligaments Disruption & & & & 3 \\
\hline Previous Implant Damage & & & & 2 \\
\hline
\end{tabular}

Table S1: Description of Every Achilles Tendon Injuries Noted on Diagnostic Ultrasound

*The total number of lesions analysed does not correspond to the number of patients included in this study. One patient may have had injuries to multiples components of the Achilles tendon which may have been described using diverse characteristics. 


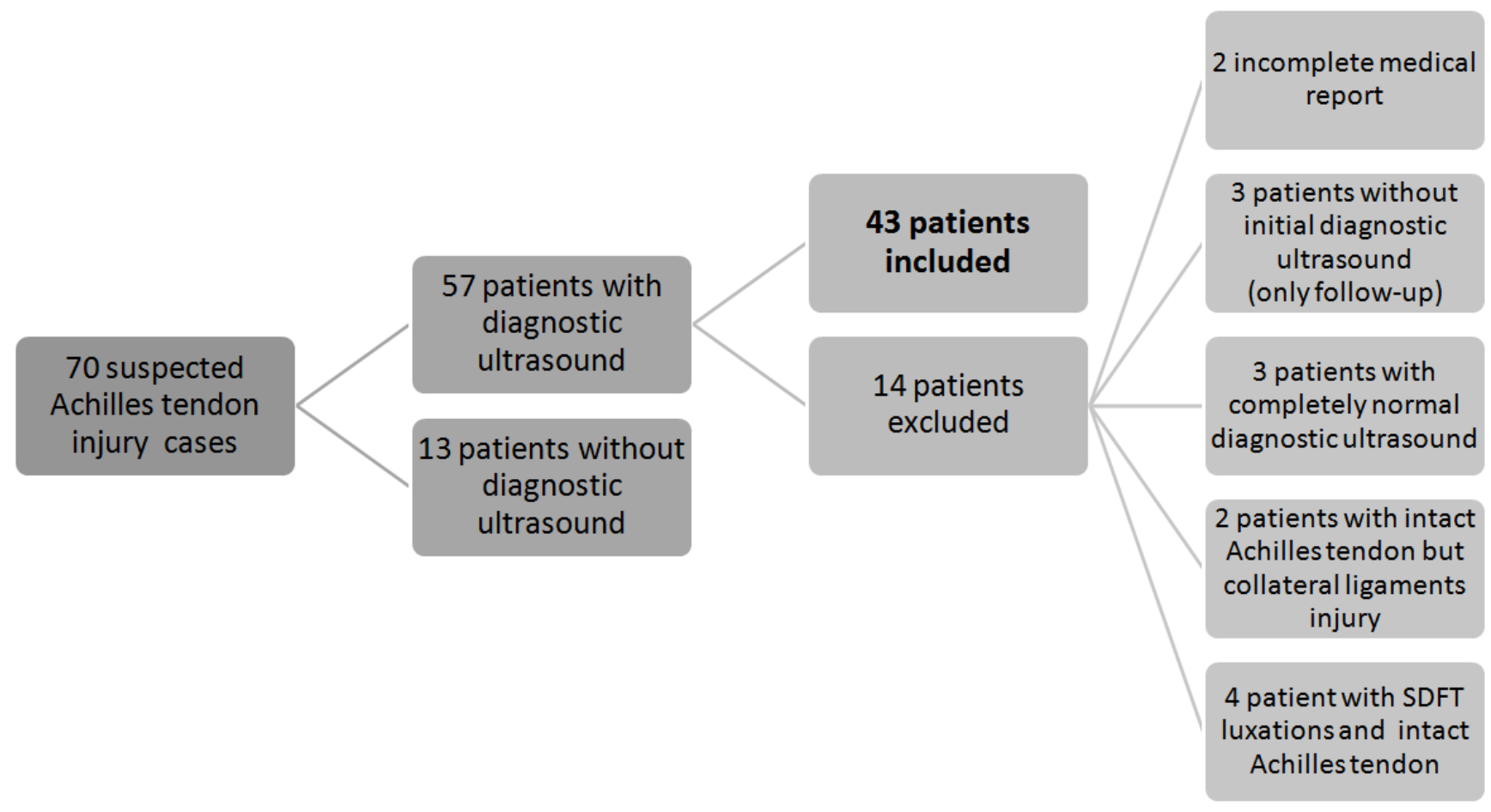

Figure S1: Organigram of the Case Selection

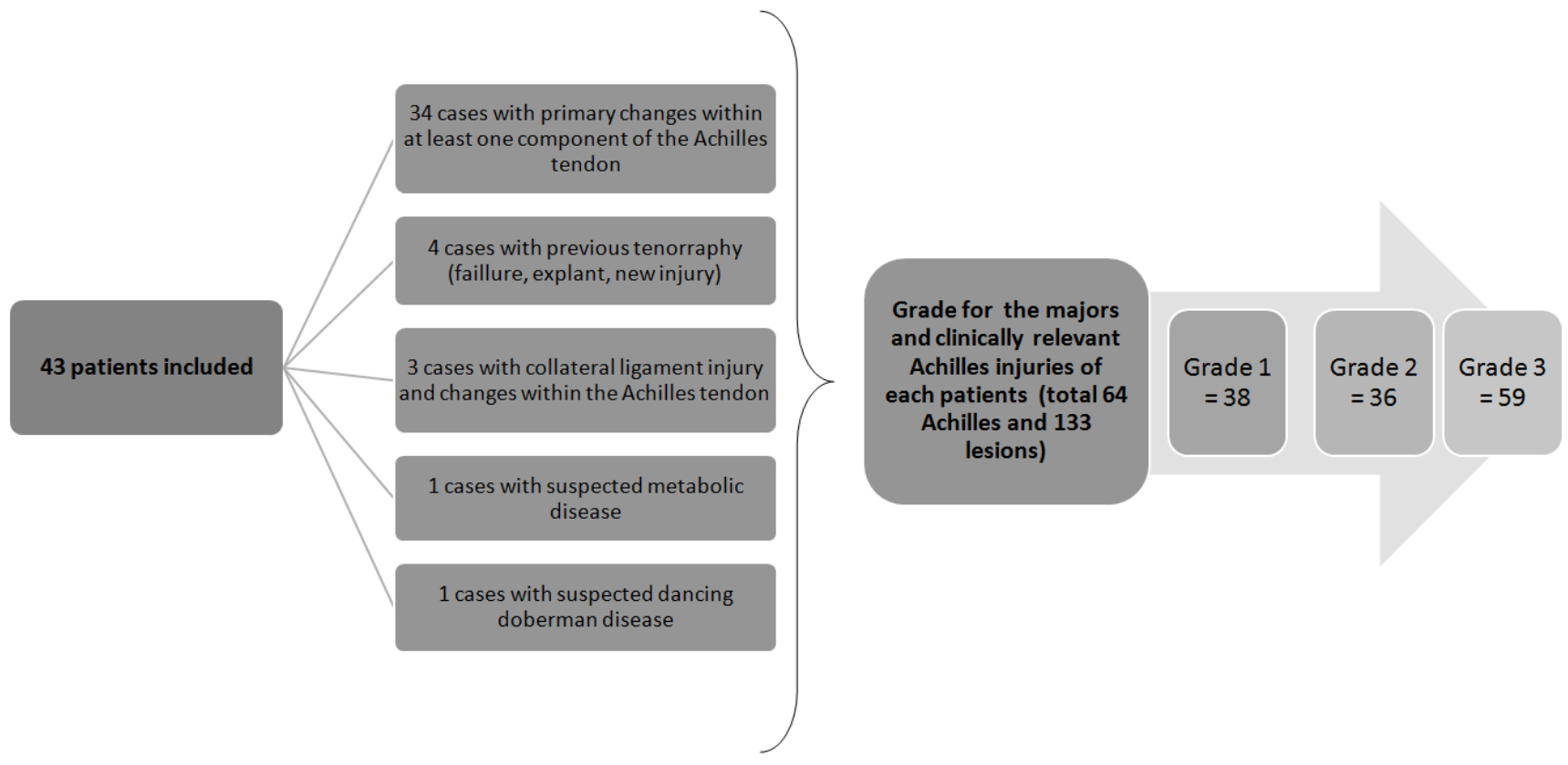

Figure S2: Organigram of the Patients Included Based on the Diagnostic Ultrasound Results

*The number of lesions analysed within each grading categories of strain is not additive and does not correspond to the number of patients included in this study. One patient may have had injuries to multiples components of the Achilles tendon which were graded separately and changes may have been bilateral. 43 patients were included in this study, 64 Achilles tendons had ultrasonic abnormalities but a total of 133 lesions were graded. 
The population reported in this case series was similar to previous studies, as Labradors and Dobermans were overrepresented (Reinke et al., 1993; Meutstege, 1993; Rivers, 1997; Kramer et al., 2001; Harasen, 2006; Corr et al., 2010). Middle-age, medium to large breed dogs seemed more predisposed to Achilles strain. Females had the tendency to be more susceptible to Achilles tendon injury, but this distinction was not significant. This finding needs to be taken cautiously due to the small sample size enrolled in this retrospective case study. A total of 130 cases of Achilles tendon injuries in five previous studies: Kramer: 20 males, 22 females Worth: 3 males, 7 females Nielsen: 18 males, 10 females Baltzer: 3 males, 2 females Corr: 22 males, 23 females (Kramer et al., 2001; Worth et al., 2004; Nielsen et al., 2006; Baltzer et al., 2009; Corr et al., 2010) identified 64 females and 66 males. With previous studies demonstrating overall equal representation of female and male, it is unlikely that any sex predilection exists in dogs. This is an indirect comparison to humans where men outnumber women with Achilles tendon injury (Silbernagel, 2015; Nyyssonen et al., 2008; Ganestam et al., 2016; Claessen et al., 2014).

The level of activity did not seem to predispose dogs to Achilles tendon injury in this study. The ten dogs with reported occupation were not more prone to a higher grade of Achilles tendon strain or complete rupture. This also seems to differ from injury in humans as it has been suggested that differences between men and women's Achilles tendon injuries might be due to different levels of the sport activities, especially ball-games (Nyyssonen et al., 2008). The authors would like to specify that the occupation was listed in the medical report; some patients may have been more active than noted within their file. The level of activity was not confirmed with the owners in this retrospective case study.

Patients with plantigrade stance on physical examination did not necessarily have a complete Achilles tear on diagnostic ultrasound. Three of the four dogs with reported plantigrade stance on physical examination were found to have a grade III strain of the GT and CT but a grade II strain of the SDFT whereas the fourth dog had a grade III strain of all Achilles tendon components. As previously reported, a plantigrade posture was not predictive of involvement of specific tendons of the Achilles tendon nor related to the grade of the strain (Corr et al., 2010). Corr concluded that plantigrade stance was more likely to be observed in animals that had suffered an injury at the musculotendinous junction, which was also observed in our study. Thus, physical examination alone may not be sufficient to characterise Achilles tendon strain. Moreover, 12 patients were subjectively exhibiting clinical evidence of unilateral strain where ultrasonic evidence of grade I-III strains was found on the contralateral Achilles tendon. The ultrasound was able to detect chronic grade I strain on the contralateral limb of 7 of these 12 dogs, and a few clinically more relevant grade II and III strains were also noted in the remaining 5 dogs. The authors believe this may be secondary to the increased sensitivity of the diagnostic ultrasound compared to the physical examination. Moreover, in 4 cases (of the 21 dogs noted to have changes within all 3 components of the Achilles tendon), the SDFT was still intact but the fibres were severely affected at the same level of the complete disruption of the GT and CT which brought up the concern for progressive break down and eventual complete rupture of the Achilles mechanism. In those cases, ultrasonography findings were essential in determining adequate treatment options. Consequently, the physical examination alone is not always sufficient to correctly assess an Achilles tendon strain and elaborate treatment recommendations; particularly with regards to individual tendon involvement, severity of strain, and laterality of the disease process.

Notwithstanding, ultrasonic changes noted within the Achilles tendon may not always be clinically relevant and may be strictly incidental. This may be the case for the previously mentioned 7 dogs that presented for unilateral pelvic limb lameness and for which grade I Achilles tendon strains were detected on the 
contralateral limb during ultrasonography. As previously reported, advanced imaging findings need to be interpreted with the clinical assessment of the patient (Rivers, 1997). Further research needs to be conducted to evaluate the clinical implication of low grade strain (grade I) and chronic changes noted on the contralateral Achilles tendon as these changes may strictly be compensatory and may not require further treatment if the primary lesion is treated on the most affected side. Measurement of each component of the Achilles tendon could also potentially help characterise the nature and severity of strain as well as objectively establish guidelines for treatment recommendations. However, measurements were not collected in this study. It has been reported that normal GT, CT and SDFT each measured 2.4-3.2 $\mathrm{mm}$ thick at mid-calcaneal tendon in crosssectional view (Lamb et al., 2005), but this has not been yet investigated in injured Achilles tendon.

Achilles injury occurs anywhere along the tendon, from the MTJ through the insertion, but more commonly occurs at/or approaching the insertion. Diagnostic ultrasound findings correlated well with surgical findings (except for one case where progression to a higher strain grade was noted from the initial diagnostic ultrasound to the surgical procedure). The sensitivity and specificity of sonographic evaluation of calcaneal tendon injury in humans has been initially reported as 0.72 and 0.83 , respectively (Kainberger et al., 1990). The Kainberger study also reported that the chronicity of Achilles tendon tears might impact the sensitivity of diagnostic ultrasound because fibrous scarring and granulomatous tissue can mask the defect. Therefore, this could lead to a false negative ultrasonographic study in chronic cases (Kainberger et al., 1990). In that study, ultrasonographic examination was performed with a $7.5 \mathrm{MHz}$ where optimal tendon sonographic evaluation is achieved with utilisation of an 10-22 MHZ transducer (Griffith, 2014). It was later reported that the use of ultrasonography is reliable in diagnosing partial ruptures of Achilles tendon with a sensitivity of 0.94, a specificity of 1.00, and an overall accuracy of 0.95 (Kälebo et al., 1992). More recent studies in humans suggest that musculoskeletal ultrasound has moderate to high diagnostic values in elucidation of Achilles tendinopathy (Henderson et al., 2015). Such data are not yet available in veterinary medicine. In the authors' opinion, the use of diagnostic ultrasound is highly dependent on the experience of the ultrasonographer, the ultrasound machine, and the knowledge of the anatomy, but can provide insight during assessment of canine Achilles tendon injury.

To the authors' knowledge, even though there have been publications describing characteristics of the normal (Lamb et al., 2005; Kramer et al., 1997; Caine et al., 2009)' and injured (Rivers, 1997; Cook, 2016; Kramer et al., 2001) Achilles tendon by diagnostic ultrasound, there has been no previous in-depth ultrasonic evaluation and characterisation of Achilles tendon strains. Despite limited sample size, the number of patients included in this retrospective study was judged sufficient to describe the most common ultrasonic findings, localisation and grading associated with Achilles tendon injury. Further study on the utility of ultrasonic assessment, especially in regards to monitoring of lesions through the treatment and recovery process, is warranted. Diagnostic ultrasound recheck examinations can potentially be a key component in assessing the structural integrity of the Achilles tendon during the treatment process. Further investigation is also needed to evaluate the effectiveness of both non-surgical and surgical treatment modalities, to establish treatment protocols based on a grading scheme for tendinous strain injury, and to evaluate the short- and long-term clinical outcome of patients. It is widely thought, that grade I and grade II strains can be managed conservatively with regenerative medicine, physical rehabilitation, and immobilisation of the tarsus joint in extension. However, surgical correction has been recommended for higher-grade injury such as grade III strains, avulsions or lacerations of the Achilles tendon. As grading of the strain help establish appropriate treatment, and since physical examination and radiographs are not sufficient to establish grading, this exemplifies the value of diagnostic musculoskeletal ultrasounds for Achilles tendon strain in dogs. 
${ }^{a}$ SoundEklin, Sound ${ }^{\mathrm{TM}}$, Carlsbad, CA, USA.

${ }^{\mathrm{b}}$ Hitachi-Aloka, Aloka, Wallingford, CO, USA.

${ }^{\text {c}}$ Sono Site, Fujifilm SonoSite, Inc., Bothell, Washington, USA.

${ }^{\mathrm{d}}$ GraphPad Prism version 6.00, GraphPad Software, La Jolla, California USA

\section{CONFLICT OF INTEREST}

Acknowledgments: The authors would like to thank Britt J. Carr, DVM, CCRT, Jennifer G. Barrett, DMV, DACVS-LA, DACVSMR, and Jennifer Brown, DMV, DACVS-LA, DACVSMR, CCRT for their help obtaining ultrasonographic imaging. Furthermore, the authors wish to thank two anonymous reviewers for their helpful and constructive comments which we believe have greatly improved the quality of this manuscript.

Funding: None

Competing interests: The authors certify that they have no affiliations with or involvement in any organization or entity with any financial interest, or non-financial interest in the subject matter or materials discussed in this manuscript.

\section{REFERENCES}

1. Archambault, J. M., Wiley, J. P., Bray, R. C., Verhoef, M., Wiseman, D. A., \& Elliott, P. D. (1998). Can sonography predict the outcome in patients with achillodynia? Journal of Clinical Ultrasound: JCU, 26(7), 335-9. http://dx.doi.org/10.1002/(SICI)1097-0096(199809)26:7\%3C335::AIDJCU1\%3E3.0.CO;2-A

2. Baltzer, W.I. \& Rist, P., 2009. Achilles Tendon Repair in Dogs Using the Semitendinosus Muscle: Surgical Technique and Short-Term Outcome in Five Dogs. Veterinary Surgery, 38(6), pp.770779. http://dx.doi.org/10.1111/j.1532-950X.2009.00565.x

3. Caine, A. et al., 2009. Sonography of the soft tissue structures of the canine tarsus. Veterinary Radiology and Ultrasound, 50(3), pp.304-308. http://dx.doi.org/10.1111/i.1740-8261.2009.01539.x

4. Case, J.B. et al., 2013. Gastrocnemius Tendon Strain in a Dog Treated With Autologous Mesenchymal Stem Cells and a Custom Orthosis. Veterinary Surgery, 42(4), pp.355360. http://dx.doi.org/10.1111/j.1532-950X.2013.12007.x

5. Cervi, M., Brebner, N. \& Liptak, J., 2010. Short- and long-term outcomes of primary achilles tendon repair in cats: 21 cases. Veterinary and Comparative Orthopaedics and Traumatology, 23(5), pp.348353. http://dx.doi.org/10.3415/VCOT-09-10-0109

6. Claessen, F.M. et al., 2014. Predictors of primary Achilles tendon ruptures. Sports medicine (Auckland, N.Z.), 44(9), pp.1241-1259. http://dx.doi.org/10.1007/s40279-014-0200-z

7. Cook, C.R., 2016. Ultrasound Imaging of the Musculoskeletal System. Veterinary Clinics of North America - Small Animal Practice, 46(3), pp.355-371. http://dx.doi.org/10.1016/j.cvsm.2015.12.001

8. Corr, S. a et al., 2010. Retrospective study of Achilles mechanism disruption in 45 dogs. Veterinary Record, 167, pp.407-411. http://dx.doi.org/10.1136/vr.c4190

9. Evans, H.E. \& De Lahunta, A., 2013. Miller's Anatomy Of The Dog (4th edition) Elsevier H., St Louis, Missouri.

10. Ganestam, A. et al., 2015. Increasing incidence of acute Achilles tendon rupture and a noticeable decline in surgical treatment from 1994 to 2013. A nationwide registry study of 33,160 patients. Knee 
Surgery, Sports Traumatology, Arthroscopy, 24(12) http://dx.doi.org/10.1007/s00167-015-3544-5

11. Griffith, J.F., 2014. Diagnostic Ultrasound: Musculoskeletal AMIRSYS; 1 Har/Psc edition

12. Harasen, G., 2006. Ruptures of the common calcaneal tendon. Canadian Veterinary Journal, 47(12), pp.1219-1220.

13. Henderson, R.E.A., Walker, B.F. \& Young, K.J., 2015. The accuracy of diagnostic ultrasound imaging for musculoskeletal soft tissue pathology of the extremities: a comprehensive review of the literature. Chiropractic \& manual therapies, 23(1), pp.31. http://dx.doi.org/10.1186/s12998-015-0076$\underline{5}$

14. Hossain, M.A. et al., 2008. Dexamethasone induces apoptosis in proliferative canine tendon cells and chondrocytes. Veterinary and Comparative Orthopaedics and Traumatology, 21(4), pp.337342. http://dx.doi.org/10.3415/VCOT-07-06-0060

15. Johnson, A., 2005. Achilles Tendon Repair. In Atlas of Orthopedic Surgical Procedures of the Dog and Cat, pp. 74-77. http://dx.doi.org/10.1016/B978-0-7216-9381-1.50028-4

16. Kainberger, F. et al., 1990. Injury of the Achilles tendon: diagnosis with sonography. American Journal of Roentgenology, 155(5), pp.1031-1036. http://dx.doi.org/10.2214/ajr.155.5.2120931

17. Kälebo, P. et al 1992. Diagnostic value of ultrasonography in partial ruptures of the Achilles tendon. Am J Sports Med., 20(4), pp.378-381. http://dx.doi.org/10.1177/036354659202000402

18. King, M. \& Jeram, R., 2003. Achilles tendon rupture in dogs. Compendium On Continuing Education For The Practicing Veterinarian, 25(8), pp.613-620.

19. Kramer, M. et al., 1998. Diseases of the achilles tendon in dogs and cats. Tierarzliche Praxis Ausgabe Kleintiere Heimtiere, 26(4), pp.238-246. [Article in German]

20. Kramer, M. et al., 1997. Sonography of the musculoskeletal system in dogs and cats. Veterinary radiology \& ultrasound : the official journal of the American College of Veterinary Radiology and the International Veterinary Radiology Association, 38(2), pp.139-149. http://dx.doi.org/10.1111/j.17408261.1997.tb00829.x

21. Kramer, M. et al., 2001. Ultrasonographic examination of injuries to the achilles tendon in dogs and cats. The Journal of small animal practice, 42(11), pp.531-535. http://dx.doi.org/10.1111/j.17485827.2001.tb06022.x

22. Lamb, C.R. \& Duvernois, A., 2005. Ultrasonographic anatomy of the normal canine calcaneal tendon. Veterinary Radiology and Ultrasound, 46(4), pp.326-330. http://dx.doi.org/10.1111/i.17408261.2005.00061.x

23. Lim, S. et al., 2008. The effects of enrofloxacin on canine tendon cells and chondrocytes proliferation in vitro. Veterinary Research Communications, 32(3), pp.243-253. http://dx.doi.org/10.1007/s11259$\underline{007-9024-8}$

24. Luscombe, K.L., Sharma, P. \& Maffulli, N., 2003. Achilles tendinopathy. Trauma, 7, pp.215225. http://dx.doi.org/10.1191/1460408603ta285oa

25. Maffulli, N., Sharma, P. \& Luscombe, K.L., 2004. Achilles tendinopathy: aetiology and management. Journal of the Royal Society of Medicine, 97(10), pp.472-476. 1258/jrsm.97.10.472

26. Meutstege, F., 1993. The classification of canine Achilles tendon lesions. Veterinary and Comparative Orthopaedics and Traumatology, 6, pp.53-55.

27. Millis DL, Levine D, Taylor RA. Canine Rehabili- tation and Physical Therapy. 1st edition. Elsevier; 2004. p. 182.

28. Morton, M. et al., 2015. Repair of chronic rupture of the insertion of the gastrocnemius tendon in the dog using a polyethylene terephthalate implant. Early clinical experience and outcome. Veterinary and Comparative Orthopaedics and Traumatology, 28, pp.282-287. doi: 10.3415/VCOT-14-08-0133.

29. Mueller, M.C. et al., 2009. Conservative treatment of partial gastrocnemius muscle avulsions in dogs using therapeutic ultrasound - A force plate study. Veterinary and Comparative Orthopaedics and Traumatology, 22(3), pp.243-248. doi: 10.3415/VCOT-08-07-0059.

30. Mughannam, A. \& Reinke, J., 1994. Avulsion of the gastrocnemius tendon in 3 cats. Journal of the American Animal Hospital Association, 30(6), pp.550-556.

31. Nielsen, C. \& Pluhar, G.E., 2006. Outcome following surgical repair of achilles tendon rupture and 
comparison between postoperative tibiotarsal immobilization methods in dogs: 28 cases (19972004). Veterinary and Comparative Orthopaedics and Traumatology, 19(4), pp.246-254.

32. Nyyssonen T. et al., 2008. The increasing incidence and difference in sex distribution of Achilles tendon rupture in Finland in 1987-1999. Scand J Surg, 97(3), pp.272275. http://dx.doi.org/10.1177/145749690809700312

33. Reinke, J., Mughannam, A. \& Owens, J., 1993. Avulsion of the gastrocnemius tendon in 11 dogs. Journal of the American Animal Hospital Association, 29(5), pp.410-418.

34. Rivers, B., et al, 1997. Sonographic findings in canine common calcaneal tendon injury. Veterinary and Comparative Orthopaedics and Traumatology , 10(1), pp.45-53.

35. Royall, N. a, 2011. Ultrasound-assisted musculoskeletal procedures: A practical overview of current literature. World Journal of Orthopedics, 2(7), pp.57-66. http://dx.doi.org/10.5312/wjo.v2.i7.57

36. Shakibaei, M. et al., 2001. Biochemical changes in Achilles tendon from juvenile dogs after treatment with ciprofloxacin or feeding a magnesium-deficient diet. Archives of Toxicology, 75(6), pp.369374. http://dx.doi.org/10.1007/s002040100243

37. Silbernagel, K.G., 2015. Sex differences in outcome after an acute achilles tendon. Orthop J Sports Med, 29(3), pp.6. doi: 10.1177/2325967115586768.

38. Spinella, G. et al., 2010. Surgical repair of Achilles tendon rupture in dogs : a review of the literature, a case report and new perspectives. Veterinarni Medicina, 55(7), pp.303-310.

39. Takebayashi, S., Takasawa, H., Banzai, Y., Miki, H., Sasaki, R., Itoh, Y., \& Matsubara, S. (1995). Sonographic findings in muscle strain injury: clinical and MR imaging correlation. Journal of Ultrasound in Medicine, 14(12), 899-905. http://dx.doi.org/10.7863/jum.1995.14.12.899

40. Vaughan, L.C., 1981. The use of carbon fibre implants for the repair of Achilles tendon rupture in dogs. The Journal of small animal practice, 22(10), pp.629-634. http://dx.doi.org/10.1111/j.17485827.1981.tb00565.x

41. Worth, A.J. et al., 2004. Ability to work and owner satisfaction following surgical repair of common calcanean tendon injuries in working dogs in New Zealand. New Zealand veterinary journal, 52(May 2015), pp.109-116. http://dx.doi.org/10.1080/00480169.2004.36415 


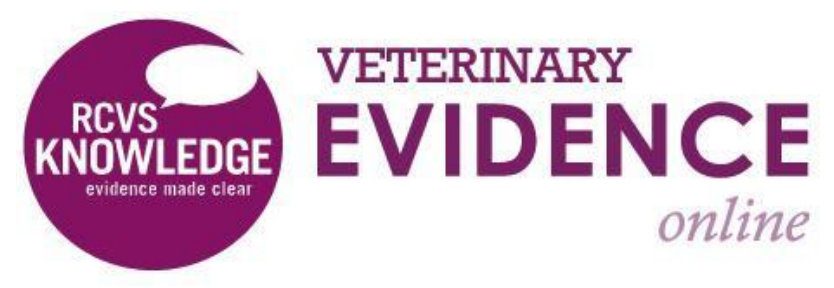

\begin{abstract}
Intellectual Property Rights
Authors of Articles submitted to RCVS Knowledge for publication will retain copyright

in their work, and will be required to grant to RCVS Knowledge a non-exclusive

license of the rights of copyright in the materials including but not limited to the right to publish, re-publish, transmit, sell, distribute and otherwise use the materials in all languages and all media throughout the world, and to license or permit others to do
\end{abstract}

so.

Veterinary Evidence and EBVM Network are RCVS Knowledge initiatives. For more information please contact us at editor@veterinaryevidence.org.

RCVS Knowledge is the independent charity associated with the Royal College of Veterinary Surgeons (RCVS). Our ambition is to become a global intermediary for evidence based veterinary knowledge by providing access to information that is of immediate value to practicing veterinary professionals and directly contributes to evidence based clinical decision-making.

www.veterinaryevidence.org

RCVS Knowledge is a registered Charity No. 230886. Registered as a Company limited by guarantee in England and Wales No. 598443.

Registered Office: Belgravia House 62-64 Horseferry Road London SW1P 2AF 